\title{
El diseño curricular en la educación técnico profesional Cubana: características teórico - metodológicas esenciales
}

\section{The curricular design in the Cuban professional technical education: theoretical - methodological essential characteristics}

\author{
Yoesdely del Carmen Cruz Acosta
}

Universidad de Camagüey, Cuba.

Enrique Aurelio Barrios Queipo

Universidad Internacional SEK, Ecuador

Autor por correspondencia: yoesdely.cruz@reduc.edu.cu, enrique.barrios@uisek.edu.ec Fecha de recepción: 7 de Febrero de 2017 - Fecha de aceptación: 28 de Abril de 2017

\section{Resumen}

La formación de técnicos medios y obreros calificados dentro del subsistema de la Educación Técnico y Profesional en Cuba, se ha caracterizado en toda su existencia por constantes cambios en su diseño curricular en la búsqueda de mejores resultados en el impacto de sus egresados en la transformación del objeto de trabajo de sus especialidades sin alcanzar tal propósito. Caracterizar el proceso de diseño curricular, y en particular el microdiseño de las asignaturas del ciclo de formación profesional técnica, permite establecer las bases para implementar acciones que eleven la estabilidad en el tiempo de sus planes y programas de estudio sus asignaturas, cuestión esta que se pretende en el presente artículo.

Palabras clave: educación técnico y profesional; diseño curricular; microdiseño curricular; ciclos formativos

\begin{abstract}
The formation of skilled technicians and skilled workers within the subsystem of Technical and Professional Education in Cuba has been characterized in all its existence by constant changes in its curricular design in the search for better results in the impact of its graduates in the Transformation of the work object of their specialties without achieving such purpose. To characterize the curricular design process, and in particular the micro-design of the subjects of the technical vocational training cycle, allows to establish the bases to implement actions that increase the stability in the time of its plans and programs of study its asignaturas, question that is Intended in this article.
\end{abstract}

Key words: technical and professional education; curricular design; curricular micro-design; training cycles 


\section{Introducción}

La Educación Técnica y Profesional (ETP), como subsistema educacional dentro del sistema nacional de educación en Cuba, tiene la finalidad de asegurar la formación de la fuerza de trabajo calificada que requiere el sector de la producción y los servicios, tanto estatal como no estatal, para el desarrollo de la economía del país.

En este empeño, sin dejar de considerar los logros alcanzados, algunos informes de investigaciones científicas: Cejas (2001), García (2002), González (2003), Forgas (2003), Barrios (2005), Téllez (2005), Castañeda (2007), Thompson (2009), reconocen las limitaciones que presentan algunos egresados de este subsistema educacional, en cuanto al dominio de la cultura profesional técnica que les permita transformar el objeto de trabajo de la profesión correspondiente, cuestión esta que se ha tratado de resolver a partir de constantes cambios en los planes y programas de estudio aplicados.

En este sentido y asumiendo la uniformidad que existe en el diseño curricular de las diferentes especialidades de la ETP, se realizó un estudio diagnóstico en la especialidad Técnico Medio en Explotación del Transporte Ferroviario, en lo adelante ETF, en la provincia de Camagüey, el cual permitió constatar que durante el desempeño de sus egresados persisten manifestaciones como resultado de insuficiencias en dominio del contenido profesional técnico, asociado al objeto de trabajo de esta profesión en el contexto, lo que fundamentó la necesidad de explorar y diagnosticar las causas que lo originan.

Para ello se profundizó en el análisis de documentos normativos (modelo del profesional y el plan de estudio) y se identificó que el diseño curricular aplicado (por ciclos formativos y asignaturas), presenta limitaciones en cuanto a la estructura lógica que se exige a este tipo de diseño, a la función de cada uno de los ciclos, a su organización y en particular, a la precisión de los contenidos del ciclo profesional técnico y de las asignaturas que lo conforman, lo que incide de manera negativa en la posibilidad de que los egresados puedan resolver los problemas profesionales más generales y frecuentes de la profesión en el contexto, y por tanto, el logro del desempeño profesional técnico aspirado.

La valoración de los elementos expuestos evidencia la necesidad de perfeccionar el proceso formativo en la ETP, para lo cual constituyen un aporte los resultados de la actividad científica desarrollada como parte de la formación doctoral en relación con el estudio teórico y práctico del microdiseño curricular de las asignaturas del ciclo de formación profesional técnica en la especialidad ETF.

\section{El Proceso de Diseño Curricular en la ETP}

Para caracterizar el diseño curricular en la ETP es necesario adoptar una posición terminológica, ya que existen diversas interpretaciones sobre este objeto de estudio.

El diseño curricular de forma general, ha sido concebido a lo largo del tiempo y en cualquier época, como el proceso a través del cual se logran establecer las relaciones entre las necesidades sociales, la escuela y el currículo formativo bajo diversos enfoques, lo que 
presupone para la ETP la existencia de variadas interpretaciones sobre su función dentro del proceso curricular.

Desde la anterior perspectiva, el diseño curricular en la ETP ha de reflejar los aspectos culturales y las tradiciones de la sociedad en relación con la intención que tiene la formación de los obreros y técnicos, así mismo, las relaciones entre teoría y práctica en dicho proceso, lo que satisface las ideas de Kemmis (1986: 30), al plantear que "el problema central de la teoría del currículo debe ser entendido como el doble problema de las relaciones entre la teoría y la práctica, por un lado, y el de las relaciones entre educación y sociedad, por otro".

Los elementos anteriores sitúan al problema del diseño curricular en el centro de los problemas de la teoría curricular, pues tienen relación con el tipo de profesional que este subsistema debe formar y con la proyección de la escuela politécnica en el cumplimiento de su misión para favorecer el desarrollo de la sociedad.

En correspondencia con lo planteado se valora la posición epistemológica que permita, desde la definición de diseño curricular, hacer realidad sus funciones de capacitar al futuro profesional técnico; de actualizar los contenidos de enseñanza en las diferentes materias a partir de los avances de la Ciencia y la Técnica y de lograr la vinculación escuela - sociedad.

Los autores de este artículo coinciden con las ideas de Forgas (2003), Barrios (2005) y Polo (2012), quienes asumen la definición que sobre diseño curricular ofrece Fuentes (2000:5) quien plantea que "es el proceso creador mediante el cual se obtiene el currículo y que como actividad de diseño implica definir bases o criterios para el diseño, sistematizar, secuenciar, controlar y retroalimentar para que el resultado satisfaga la necesidad de su elaboración".

En la definición asumida se destacan tres elementos que han sido poco atendidos en la historia del desarrollo de la ETP cubana: su carácter creador que implica, entre otros aspectos, un mayor protagonismo de sus participantes en la sistematización que se ha de generar en cada uno de los componentes didácticos y la posibilidad de retroalimentación a que deben ser sometidos de manera continua y constante los mismos en sus niveles de concreción, cuestiones que, a diferencia de las definiciones de diseño curricular dadas por Valdés y Portuondo (1997), Álvarez C. (1999), Addine y otros (2000) y Castañeda (2007), imprimen una nueva dinámica a este proceso.

El análisis referido permite profundizar no solo en la caracterización del diseño curricular, sino también en sus relaciones más esenciales; la primera, dada entre el medio social y el proceso formativo y la segunda, de manera interna entre sus componentes.

El conocimiento científico acerca del diseño curricular está determinado por la primera ley de la Didáctica, "Relaciones del proceso docente educativo con el contexto social: La escuela en la vida", (Álvarez, C., s/f: 39). Son diversas la ideas acerca del sistema que este conocimiento determina, y cuando de la ETP se trata, generalmente se parte de la existencia de necesidades sociales que justifican la presencia de profesiones técnicas en las cuales se exige la formación de un sujeto para que resuelva, a través de su desempeño profesional, la producción de bienes materiales y de servicios. De esta manera es que queda establecido el nexo entre las profesiones 
técnicas, su proceso profesional, sus problemas profesionales, sus tareas y ocupaciones y su proceso curricular, con el encargo social de la formación de un tipo de profesional técnico, de su proceso formativo, (Figura 1).

Por su parte, la relación interna entre los componentes del diseño curricular queda establecida mediante la concreción curricular que se adopte, pudiendo encontrar en la literatura nacional y foránea dos términos para expresar esta concreción curricular: dimensiones o niveles. En esta investigación se asume el término niveles, estructurados según Addine y otros (2000), en los niveles de macro, meso y microdiseño curricular, los cuales serán abordados de manera general a continuación:

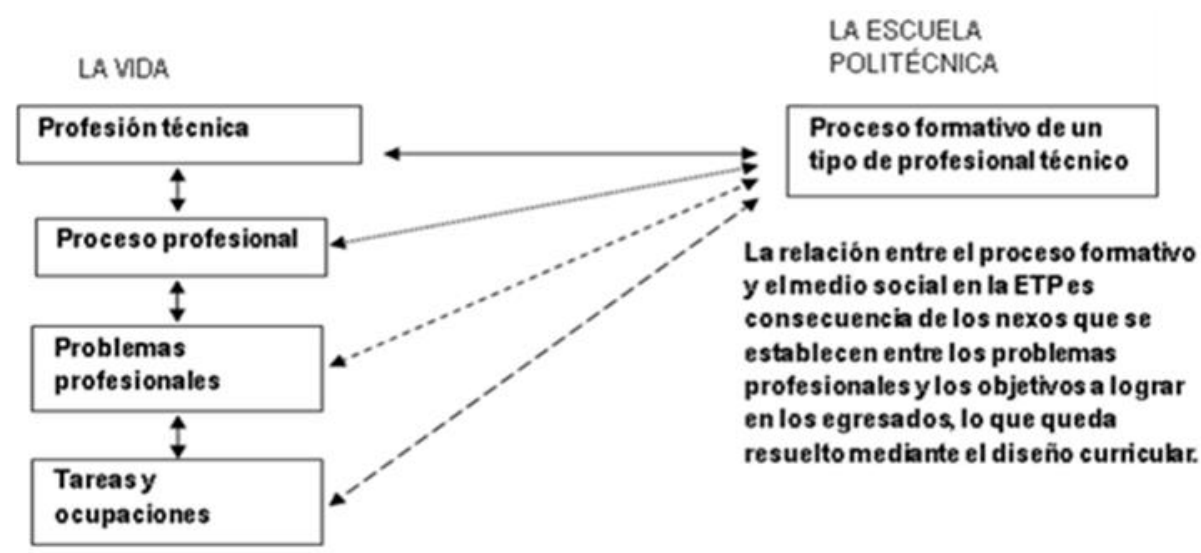

Figura 1: Vínculo entre el proceso profesional y el proceso formativo.

El primer nivel de concreción curricular (macrodiseño), señala las líneas del pensamiento educativo, de la política educacional y las metas trazadas, de manera que orienten a los siguientes niveles de concreción y al desarrollo del currículo sobre el plan de acción a seguir. Con estos elementos, coinciden los autores del presente artículo, sin embargo, consideran que no se reconoce la implicación de este proceso en la determinación de los aspectos más generales y trascendentes de la profesión, del modelo del profesional y la estructura de las especialidades, tal y como lo hace Forgas (2003), quien le da un papel más concreto a este nivel del diseño curricular al concebirlo como el "proceso que se efectúa a nivel de especialidad, y tiene como objetivo la precisión del modelo del profesional que se aspira a formar, el diseño del plan de estudio, la estructura de la especialidad caracterizado por cierto grado de flexibilidad dejando espacio a posibles ajustes, cambios y enriquecimiento de su contenido y estructura".

En la actualidad, el macrodiseño curricular en la ETP y por tanto en la especialidad ETF, es un proceso que realiza la comisión nacional de expertos del Mined y especialistas de los Organismos de la Administración Central del Estado, en el cual se obtienen como resultados fundamentales, el modelo del profesional técnico, el plan de estudio y los programas de las asignaturas.

Este modelo del profesional contiene el objeto de trabajo de la especialidad, el campo de acción, las tareas y ocupaciones, los objetivos y habilidades generales y por años, las orientaciones para el desarrollo de las prácticas pre profesionales, las conferencias técnicas, cursos de complementación y la culminación de estudios. 
Es de destacar que no se manifiesta una profunda caracterización de la profesión, ni se refieren los problemas profesionales; se limita a la identificación de las tareas y ocupaciones previstas en los cargos ocupacionales determinados por el Ministerio de Trabajo y Seguridad Social, lo cual se contrapone con el reconocimiento de que las profesiones técnicas existen porque hay problemas profesionales técnicos que requieren de un tipo de egresado que los resuelva.

Esa necesidad es expresión de la intencionalidad del proceso formativo, y son múltiples las experiencias que demuestran que el modelo del profesional técnico alcanzado desde los problemas profesionales, constituye uno de los referentes que mejor explica el vínculo de la sociedad con el centro politécnico y viceversa: Patiño (1988), Hernández (2000), García (2002), León (2003), Forgas (2003), Mena (2003), Jiménez (2003), Abreu (2004), Barrios (2005), Castañeda (2007), Mena (2008), Thompsom (2009) y Gallart (2011). Puede plantearse entonces, que este fenómeno es una de las principales limitaciones en la concreción del macrodiseño curricular actual de la ETP para alcanzar las exigencias que el proceso profesional técnico le impone al proceso formativo.

En cuanto a la concepción de los planes de estudio de la ETP, a partir de la revisión realizada, pudo comprobarse que desde 1980 presentan su estructura en ciclos formativos, los cuales según Álvarez, C. (1999), constituyen el elemento de enlace con la profesión, pues responden a la necesidad de diferenciar la génesis del objeto de la profesión, que en el caso de la ETP es de tipo profesional técnica.

Son diversas las concepciones que se utilizan para estructurar y organizar los planes de estudio con el empleo de los ciclos formativos. En la presente investigación se sistematiza el resultado de Barrios (2005), quien propone una clasificación de los ciclos a partir de su esencia y función, así como de su contenido dentro del currículo formativo, (Tabla 1).

Tabla 1: Contenido que caracteriza a los ciclo formativos en la ETP, según el modelo
de actuación del profesional técnico, Barrios (2005).
\begin{tabular}{|c|l|}
\hline $\begin{array}{c}\text { Ciclos del plan de } \\
\text { estudio }\end{array}$ & \multicolumn{1}{c|}{ Contenido que los caracteriza } \\
\hline Formación general & Objetos de la cultura que tienden a cualificar el modo de actuar del técnico. \\
\hline Básico técnico & $\begin{array}{l}\text { Objeto de la cultura que modela a los objetos naturales, cuyas leyes fueron } \\
\text { empleadas para su transformación en objetos e instrumentos de trabajo, los } \\
\text { cuales intervienen en procesos tecnológicos especificos. }\end{array}$ \\
\hline Técnico específico & $\begin{array}{l}\text { Objetos fundamentos de las ciencias que explican los procesos, objetos e } \\
\text { instrumentos de trabajo que intervienen en los procesos tecnológicos. }\end{array}$ \\
\hline $\begin{array}{c}\text { Ejercicio de la } \\
\text { profesión técnica }\end{array}$ & $\begin{array}{l}\text { Ciencia de un proceso tecnológico particular, que constituye su objeto de } \\
\text { trabajo e interviene en procesos tecnológicos especificos. }\end{array}$ \\
\hline $\begin{array}{c}\text { Especialización de } \\
\text { la profesión técnica }\end{array}$ & $\begin{array}{l}\text { Ciencia de un proceso tecnológico especifico, que constituye su objeto de } \\
\text { trabajo, donde intervienen técnicas y procedimientos. }\end{array}$ \\
\hline
\end{tabular}

Atendiendo a que el campo de acción de la investigación se enmarca en las asignaturas de formación profesional técnica es posible agrupar a los ciclos Técnico Específico, Ejercicio de la 
Profesión y Especialización Técnica, dentro de uno denominado ciclo de formación profesional técnica (Figura 2), que resume la función de los que lo componen.

El segundo nivel de concreción del diseño curricular (meso diseño), tiene su manifestación en las instituciones educativas o instancias intermedias del sistema educacional a partir de proyectos educativos. En correspondencia con las características actuales de la ETP, resulta insuficiente el sistema que permite relacionar el carácter universal que presupone el macrodiseño curricular con los proyectos que han de generar, en lo productivo y lo económico, en las provincias y los municipios del país. Esto conduce a los autores de este artículo a solo profundizar en los niveles macro y micro curriculares.

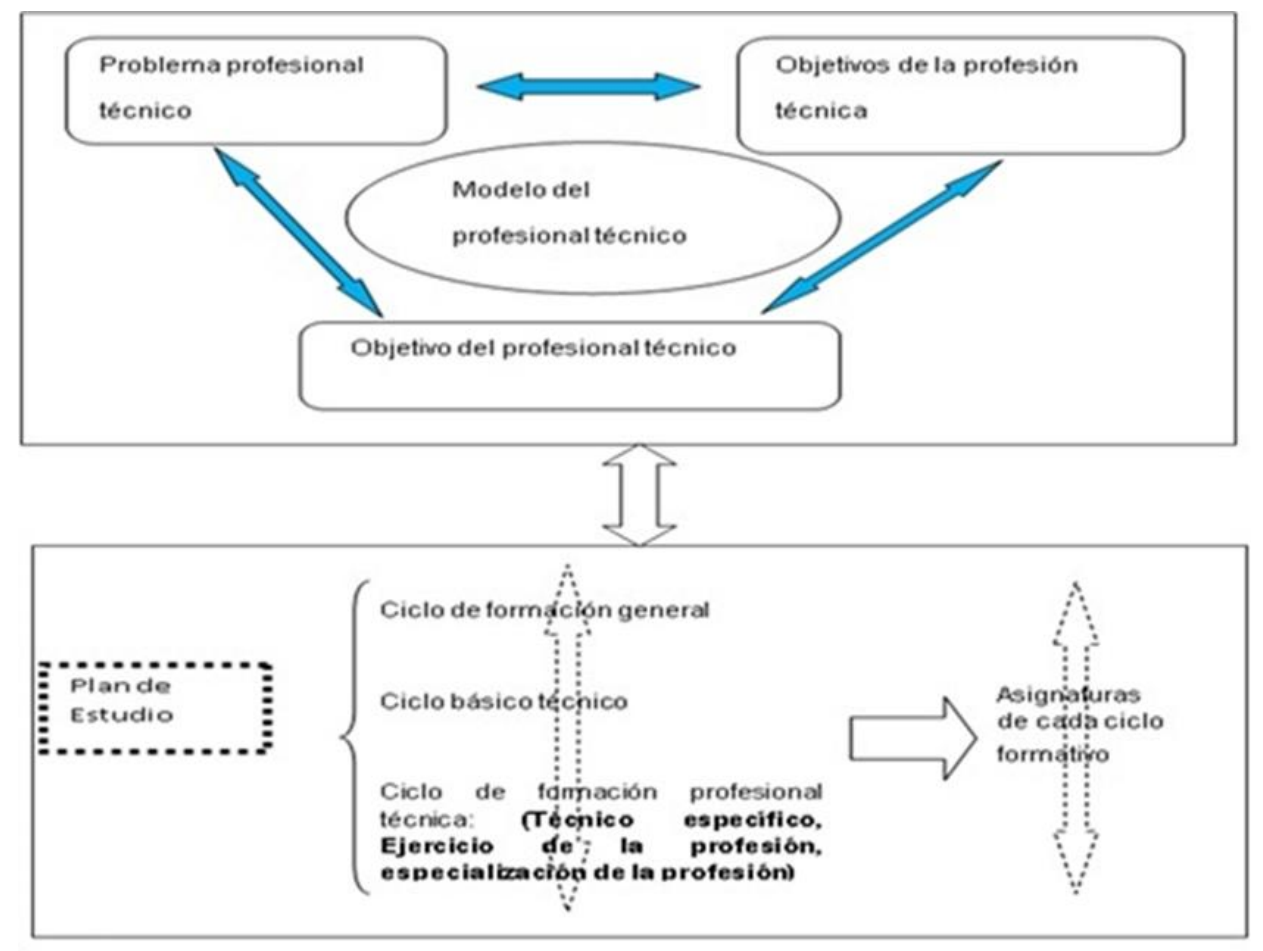

Figura 2: concepción del ciclo formativo de formación profesional técnica.

El segundo nivel de concreción del diseño curricular (mesodiseño), tiene su manifestación en las instituciones educativas o instancias intermedias del sistema educacional a partir de proyectos educativos. En correspondencia con las características actuales de la ETP, resulta insuficiente el sistema que permite relacionar el carácter universal que presupone el macrodiseño curricular con los proyectos que han de generar, en lo productivo y lo económico, en las provincias y los municipios del país. Esto conduce a los autores de este artículo a solo profundizar en los niveles macro y micro curriculares.

En el tercer nivel de concreción del diseño curricular (microdiseño) se determinan los objetivos didácticos, contenidos, actividades de desarrollo, actividades de evaluación y la metodología que cada área materializará en el aula, en aras de precisar el contenido curricular, que es su esencia, lo cual debe lograrse dentro de un proceso creativo que sistematice el contenido en estrecho vínculo con el contexto para promover una constante retroalimentación. 
Por la importancia que tiene este nivel de concreción del diseño curricular para la investigación que se presenta, será analizado con mayor profundidad en el epígrafe siguiente.

\section{El Proceso de Microdiseño Curricular en la ETP Cubana.}

En el modelo del profesional, como resultado del macrodiseño curricular, se determinan los aspectos más generales y trascendentes que deben ser incorporados al proceso de formación de los profesionales técnicos con una intencionalidad formativa que debe incidir de forma directa en el desempeño profesional y social de los egresados, lo que debe quedar precisado durante el microdiseño curricular.

En el microdiseño curricular, según Álvarez, R. (1987), las características de la época y en particular, de la micro sociedad, son las que marcan pautas para la obtención del currículo. Esta aseveración fundamenta la necesidad de concebir las asignaturas en función del denominado desarrollo contextual.

El desarrollo contextual en Cuba, también reconocido en la literatura consultada como desarrollo local (Affonso y Rui de Britto (2000), Alburquerque (2004), Guzón y otros (2011), León y otros (2011), Alonso (2012)), es parte de la política de la Revolución y se articula de manera planificada a la estrategia de desarrollo nacional. Constituye un proceso multidimensional que se concreta en estrategias municipales elaboradas y llevadas a la práctica dentro de un clima de cooperación entre todos los factores que garantizan dicho desarrollo, donde el papel de la educación en general y de la ETP en particular es determinante.

Para alcanzar las pautas en la obtención del currículo dentro del propósito referenciado y en atención a la teoría de diseño curricular que se asume, es necesario superar las deficiencias que presentan los planes de estudio de la ETP, en cuanto a la falta de precisión de los métodos y procedimientos asociados a los paradigmas tecnológicos contextuales.

El vocablo paradigma proviene del griego, entendiéndose como ejemplo que sirve de norma. Se identifica también con "principios que asocian o disocian las nociones fundamentales que rigen y controlan todo el discurso teórico que transforma la realidad, lo cual significa que una modificación en el paradigma trae consigo la modificación de la realidad", Rosental y Ludin (1981:34).

Un paradigma, según De la Herrán, Hashimoto y Machado (2004), es un logro científico fundamental que incluye una teoría, aplicaciones ejemplares, plantea tareas abiertas, así como la creencia aceptada por un grupo de que no hay que polemizar con ese paradigma sino aplicarlo, explotarlo.

Para Barrios (2005), una tecnología específica está relacionada con un paradigma tecnológico concreto, el cual está conformado por un conjunto de reglas o principios que guían las decisiones de un proceso tecnológico, asociadas al conocimiento científico de la profesión correspondiente y quedan expresadas, en el caso de los profesionales técnicos, mediante los 
métodos y procedimientos empleados durante la transformación del objeto de trabajo, los cuales en su condición de contenido tienen un carácter universal y contextual.

En el caso de la especialidad ETF y en consideración a las valoraciones realizadas mediante entrevistas a expertos, se pudo determinar entre otros aspectos qué métodos y procedimientos tecnológicos se presentaron en cada etapa del desarrollo ferroviario y las distintas formas de concebirlos, de lo que se concluye que:

- La ETF se sintetiza como un proceso mediante el cual se obtiene el máximo provecho y productividad a los equipos rodantes existentes y a las vías por las cuales circulan los mismos, lo que puede quedar representado a través de la tríada que se muestra en la figura 3.

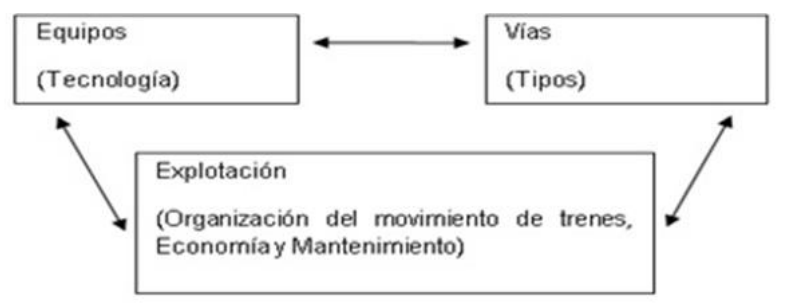

Figura 3: Triada que sintetiza la función de explotación del transporte ferroviario.

Los paradigmas que han caracterizado de manera global las tecnologías, según Pérez (2004), y que tienen repercusión en la evolución de la ETF son: la época de hierro (1829 - 1875); la época del acero (1875 - 1808); la época del petróleo (1908 - 1975); hasta la actual época de la informática y las telecomunicaciones.

En Cuba, la explotación del sector ferroviario data de 1837. Los paradigmas tecnológicos señalados en el apartado anterior, se han caracterizado por la aplicación de métodos y procedimientos que inicialmente tuvieron un origen inglés, para la zona occidental del país y estadounidense, para la zona oriental, aunque también se incorporan otras, hasta nuestros días, de diferentes procedencias, lo que implica una diversidad de aplicaciones y formas de concebir dicha explotación.

- En la actualidad, prevalecen en el país diferentes métodos y procedimientos tecnológicos para la ETF. Se debe incorporar en la medida del desarrollo económico, una explotación que descanse en factores claves como la microelectrónica, las tecnologías digitales, los equipamientos de informática y telecomunicaciones, la robótica, el software y los servicios. Esta realidad justifica la necesidad de que la ETP así lo reconozca.

Otro aspecto determinante para la concreción del currículo que realizan los profesores de la ETP, en atención a Arbizu (1998), es el tipo de modelo curricular establecido, a saber: los modelos escuela, empresa o escuela-empresa. La ETP cubana aplica el modelo escuela empresa, que se caracteriza por favorecer durante la determinación del contenido curricular, la atención al principio de la integración del estudio con el trabajo, como expresión fundamental de la vinculación de la escuela con la vida. Este modelo permite combinar tareas pedagógicas y de 
producción durante el proceso formativo, lo que garantiza la formación de conocimientos, habilidades y valores propios de cada profesión.

La efectividad en la aplicación del modelo escuela - empresa en la ETP cubana requiere no solo de un proceso curricular con características innovadoras en cuanto a vías para su materialización sino también de cierta descentralización y flexibilidad desde la concepción del diseño curricular.

La innovación a que se hace referencia puede ser considerada atendiendo a varios factores, pero cuando se trata del diseño curricular desarrollador adquiere mayor relevancia, porque presupone un proceso que permanentemente se encuentra influenciado por la ejecución de dicho diseño.

De acuerdo con Díaz Barriga, F., Lule, M., Rojas, S. y Saád, S. (1990), la innovación es expresión de lo técnico y lo social, por su parte De la Torre (1996), plantea que es un proceso complejo, contextual, sistémico y global protagonizado por un colectivo concreto que trata de transformar la práctica educativa a partir del tratamiento de las necesidades que le afectan.

Teniendo en cuenta el propósito de esta investigación, se consideran tres elementos que constituyen fuente de la innovación señalada, son estos: la concepción de diseño curricular desarrollador asumida, la exigencia social de incorporar contenidos profesionales técnicos contextuales que lo retroalimenta y el efecto que causa la categoría didáctica contenido en la dinámica curricular.

En cuanto a la descentralización, ésta es una característica del diseño curricular que permite que en cada uno de sus niveles se pueda desarrollar la iniciativa y la creatividad de los sujetos participantes. Cada nivel debe evitar la pormenorización excesiva de su contenido de manera que los objetivos, sin ser imprecisos, sean lo suficientemente generales como para posibilitar diversas variantes en relación a la individualidad de sus protagonistas. De ese modo se debe elaborar el plan de estudio y el resto del currículo, lo cual incluye los programas de asignaturas. A su vez, estos programas deben permitir a quienes los desarrollen, la atención a enfoques diferentes y que en un momento determinado puedan cuestionar, incluso, la planificación general, provocando un proceso que conlleve al perfeccionamiento del currículo.

En las concepciones del modelo del profesional técnico cubano se asegura que existe flexibilidad curricular (Patiño, 1996). Esta característica, según Barrios (2005), inherente al modelo curricular debe fijar a través de su metodología curricular, la idea de que el proceso de toma de decisiones para la aplicación del currículo tenga en sus dimensiones gnoseológica, profesional y metodológica una concreción real de los postulados del modelo curricular correspondiente; idea poco probable de alcanzar en la ETP, si se tiene en cuenta que los profesores plantearon durante el diagnóstico inicial, la existencia de limitaciones en el diseño curricular, dada la incoherencia que muestra históricamente la declaración de cierta flexibilidad entre el macro y el microcurrículo, en contraposición a la rigidez de este último para incorporar al proceso formativo los métodos y procedimientos del paradigma tecnológico de la profesión técnica en el contexto. 
Atendiendo a los presupuestos señalados, en la ETP se pierde la lógica que suscitó el macrodiseño curricular durante el microdiseño, originándose una especie de inmovilidad, reproduciéndose en el tiempo iguales contenidos ante situaciones profesionales técnicas diferentes, cambiantes.

En este sentido, Fuentes y Lucio (2009) apuntan que en la Educación Superior el microdiseño curricular es la dimensión que comprende el proceso de elaboración, por parte del profesorado, de los programas de los componentes de formación, las asignaturas y los temas, donde queda expresado el proceso de tránsito de objeto de la profesión a contenido que se desarrolla en el proceso formativo en la función de precisar los contenidos formativos. Tomando en consideración el valor gnoseológico y metodológico de lo aportado por este autor para el diseño curricular en general, en la especificidad de la ETP se hace necesario profundizar en cómo modelar dicho proceso de precisión del contenido, fundamentalmente por:

a) La limitada experiencia que acumulan los profesores sobre el microdiseño curricular, dentro de un diseño curricular por ciclos formativos que establece un plan de estudio estructurado por asignaturas que se elaboran durante el macrodiseño curricular.

b) El hecho de que la formación de los profesionales técnicos transcurre desde lo fenomenológico de la tecnología y no desde su esencia, cuestión que complejiza la relación entre la funda mentalización y la profesionalización del currículo.

Para poder considerar el proceso de microdiseño curricular en la ETP en función de la precisión del contenido y como parte de la obtención del currículo, así como para satisfacer desde el punto de vista teórico el vacío que en el mismo se presenta, las definiciones consultadas sobre el mismo (Forgas (2003) y Polo (2012)), no expresan con precisión en qué verdaderamente consiste este proceso para este subsistema educativo, y en específico para el ciclo de formación profesional técnica.

En tal sentido, la interpretación que sobre el microdiseño curricular de las asignaturas del ciclo de formación profesional técnica se tiene en esta investigación, queda planteada de la siguiente manera: "proceso innovador mediante el cual se precisan los contenidos profesionales técnicos, en una síntesis de su carácter universal y a la vez contextual, como contribución a la obtención del currículo", Cruz (2012:12).

Desde el punto de vista teórico, la precisión de los contenidos profesionales técnicos, esencia del microdiseño curricular, conlleva a la sistematización del objeto de la profesión hasta que pueda expresarse en el contenido de los programas de asignaturas y sus temas, lo que no ha quedado develado en todas sus partes y lógica del diseño curricular en la ETP, quedando al descubierto que es necesario enriquecer la teoría que explique y sustente tal microdiseño y en consecuencia, ofrecer nuevas vías que metodológicamente permitan que los profesores del ciclo de formación profesional técnica puedan desarrollarlo.

En consecuencia, el vacío teórico que se deriva de lo anterior quedó determinado en la falta de fundamentación del proceso de tránsito de objeto del ciclo de formación profesional técnica a contenido de sus asignaturas. 
El proceso de tránsito de objeto del ciclo de formación profesional técnica a contenido de sus asignaturas, puede ser común para todas sus especialidades, las que según los planes de estudio vigentes alcanzan la cifra de 51 con la calificación de Técnico Medio y 46 de Obrero Calificado (Lotti, 2014). En cualquier caso, su esencia radica en la consideración de que el objeto del profesional previsto durante el macrodiseño curricular, sintetiza la cultura profesional, general y social de la que se tiene que apropiar el estudiante de la ETP a lo largo de su currículo, condensada en la concepción del modelo del profesional y el plan de estudio correspondiente, lo que debe ser revelado en el contenido docente de los diferentes niveles (Ejemplos: competencias, ciclos, módulos, disciplinas, asignatura, temas) en que se estructura cada especialidad, (Figura 4).

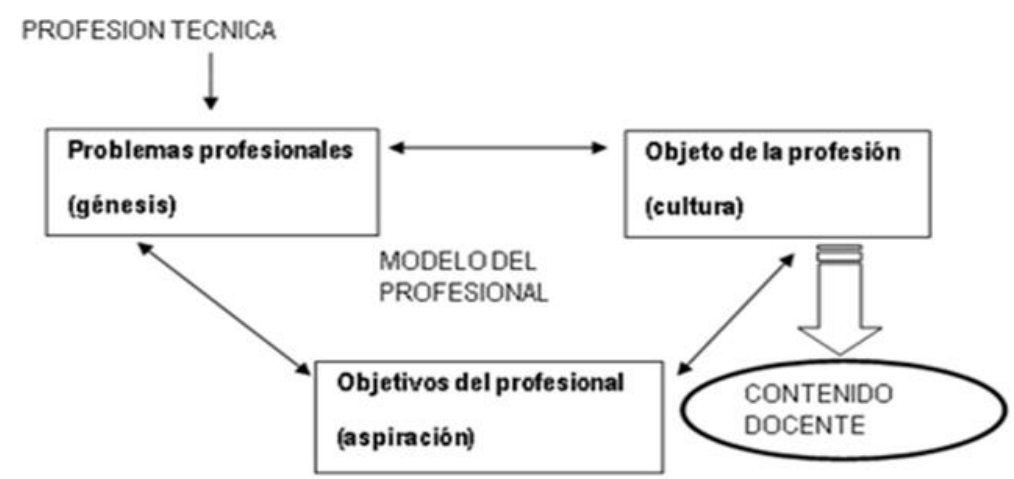

Figura 4: Génesis del contenido docente de acurdo con la concepción de diseño curricular de Fuentes y Lucio (2009).

De la cultura profesional, general y social inherente al objeto de la profesión, se seleccionan aquellos contenidos docentes que le permiten al profesional técnico resolver los problemas profesionales y poseer, además, ciertas cualidades en su personalidad desde el contenido propio de la función del ciclo de formación profesional técnico, (Figura 5).

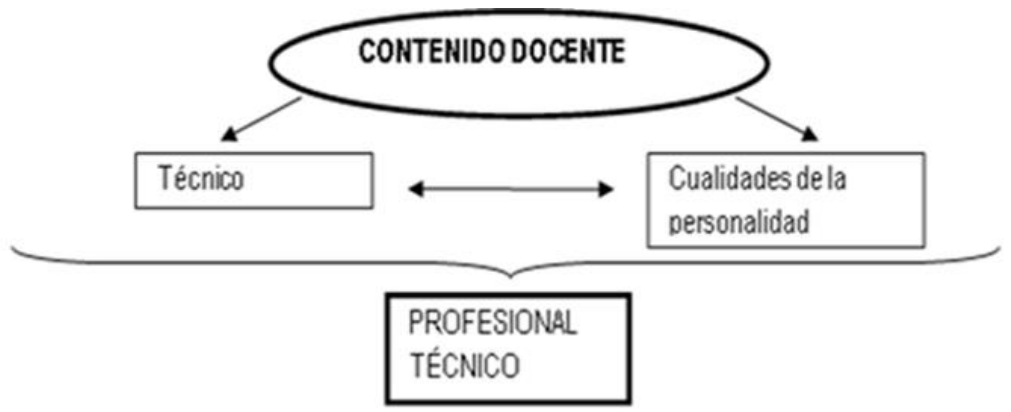

Figura 5: Caracteristica del contenido docente derivado del modelo de actuación del profesional técnico de Barrios (2005).

El proceso de tránsito referido, desde la teoría curricular que se asume, destaca el valor de la oferta formativa (a partir del diseño curricular) para satisfacer las demandas sociales (cualitativas y cuantitativas). Es importante señalar, que dicha oferta en la ETP se encuentra limitada en la consideración de aspectos trascendentales de las profesiones técnicas como la evolución de los métodos y procedimientos de los paradigmas tecnológicos, la movilidad profesional, la atención a los resultados de los estudios demográficos, entre otros, que no pueden 
obviarse porque, de lo contrario empeñaría el impacto de la fuerza de trabajo calificada por la ETP respecto al desarrollo contextual.

Sin embargo, se pudo comprobar en la sistematización realizada sobre esta temática que las relaciones entre el diseño curricular y el desarrollo contextual han sido poco estudiadas, lo que condiciona la necesidad de destacar el papel de los egresados de este subsistema educacional en la actualización del modelo socioeconómico cubano y la potencialidad que tienen las demandas del contexto en la génesis del diseño curricular de cualquier especialidad.

Para la presente investigación el desarrollo contextual vinculado con el impacto que promueven los egresados de la especialidad ETF, se asocia a procesos tales como el crecimiento de la producción (planes de transportación de cargas y pasajeros), el avance científico técnico (incorporación de nuevas tecnologías e infraestructura), la distribución de las oportunidades individuales y colectivas, el cuidado de los recursos, del medio ambiente y la organización territorial de la sociedad, para garantizar el cumplimiento del objetivo del sistema nacional de transporte ferroviario, a partir de la explotación adecuada de este importante medio de transporte en dicho contexto.

El diseño curricular en la especialidad ETF en función de satisfacer la necesidad que justifica su elaboración, ha de responder a la relación que expresa el vínculo entre el centro politécnico y la sociedad, condicionante que presupone definir las características más esenciales del egresado de la ETP, expresadas en los aspectos más generales y trascendentes que deben ser incorporados al proceso de formación de los profesionales técnicos. Este resultado alcanza una significación adicional, cuando se tiene en consideración que las características de la formación de este tipo de profesional técnico en la ETP debe ser universal y a la vez contextual, comprometida con una realidad social y profesional que se hace más compleja en medio del proceso actual de reanimación del ferrocarril cubano.

Una escuela politécnica y una especialidad de la ETP que no se encuentren integrados a las características y aspiraciones del contexto y de los procesos profesionales que allí tienen lugar y viceversa, no puede generar desarrollo en el contexto, tal y como demanda el modelo socioeconómico actual.

A partir de la caracterización, que en el orden teórico y metodológico se ha realizado, sobre el proceso de microdiseño curricular en la especialidad ETF puede concluirse que:

a) Los ciclos que estructuran en la actualidad los planes de estudio de la ETP no expresan la generalización formativa del contenido profesional técnico presente en el contexto.

b) La teoría que sustenta al proceso de microdiseño curricular en la ETP, no responde a las actuales exigencias de la obtención del currículo, por lo que se hizo necesario definir el concepto de microdiseño curricular de las asignaturas del ciclo de formación profesional técnica, si desde la ciencia se pretende fundamentar teórica y metodológicamente el tránsito de objeto del ciclo de formación profesional técnica a contenido de sus asignaturas.

c) La práctica curricular en la ETP, demuestra inconsistencias teóricas en cuanto a la implicación epistemológica, profesional técnica y de sistematización del contenido que se 
corresponde con los métodos y procedimientos tecnológicos del contexto, aspectos que permitirían precisar, en un proceso creativo, el contenido del ciclo de formación profesional técnica, durante la reelaboración de los programas de asignaturas, lo cual es la esencia del proceso de microdiseño curricular.

\section{Bibliografía}

Abreu, R. (2004). Modelo Teórico Básico de la Pedagogía Profesional. (Tesis doctoral inédita). ISPETP”Héctor Alfredo Pineda”, La Habana, Cuba.

Addine, F., González M., Batista, L., Pla, R., Laffita, R., Quintero, G., (...) Jiménez, S. (2000). Diseño Curricular. La Habana, Cuba, IPLAC.

Affonso, A. y Rui de Britto. (2000). Descentralizaçao, desenvolvimiento local e crise da Federaçao no Brasil. Santiago de Chile: Comisión Económica para América Latina y el Caribe (CEPAL).

Alburquerque, M. (2004) Desarrollo económico local y descentralización en América Latina. Investigación de la CEPAL. Revista de la CEPAL N 82. Abril, 2004.

Alonso, A. (2012). La Economía Regional: cambio de paradigma. Consideraciones para Cuba. (Tesis de Maestría inédita). Universidad de La Habana, La Habana, Cuba.

Álvarez de Zayas, C. (1995). Modelar lo que Investigo. Metodología de la Investigación Científica. I Parte Cómo se Modela la Investigación. Capítulo 4. Santiago de Cuba: Centro de Estudios de Educación Superior "Manuel F. Gran”.

Álvarez de Zayas, C. (1996). Hacia una escuela de excelencia. La Habana: Academia.

Álvarez de Zayas, C. (s/f) La escuela en la vida. Didáctica. Ciudad de la Habana: Pueblo y Educación.

Álvarez de Zayas, C. (1999). Pedagogía como ciencia o Epistemología de la Educación. [En línea]. La Habana: Félix Varela. Recuperado el 14 de mayo del 2009 de

Álvarez de Zayas, R. (1997). Hacia un curriculum integral y contextualizado. La Habana: Academia.

Arbizu, M. (1998). La Formación Profesional Específica. Claves para el desarrollo curricular. Madrid: Grupo Santillana de Ediciones.

Barrios, E. (2005). Modelo desarrollador de actuación del profesional técnico. (Tesis doctoral inédita). ISP “José Martí, Camagüey, Cuba.

Castañeda E. (1997). Curso de Pedagogía para profesores jóvenes. Teoría y Práctica del Diseño Curricular. La Habana: Universidad Virtual CUJAE. 
Cejas E. (2001). Formación por competencias profesionales: una experiencia cubana. Curso No. 2, Pedagogía 2001.

CEPROF. (1995). La evolución histórica de la formación profesional en Cuba, citado en Modelo de la Escuela Politécnica Cubana. (Soporte electrónico).

Cortijo, R. y otros (1994). Hacia una Pedagogía Profesional: cómo formar un trabajador competente. La Habana: ISPETP”Héctor Pineda Zaldívar”. (Soporte electrónico).

Cruz, Y. (2012). Metodología para la retroalimentación curricular en la ETP. CD-R V Taller Nacional Científico Metodológico sobre formación laboral. ISBN 978-959-18-0381-8. Holguín, 2012.

De la Herrán, G., Hashimoto, E. y Machado, E. (2004). Investigar en Educación: fundamentos, aplicación y nuevas perspectivas. Madrid.

Díaz Barriga, F., Lule, M., Rojas, S. y Saád, S. (1990). Metodología de Diseño Curricular para Educación Superior. México: Trillas.

Forgas, J. (2003). Modelo para la formación profesional, en la educación técnica y profesional, sobre la base de competencias profesionales, en la rama Mecánica. (Tesis doctoral inédita). UCP "Frank País", Santiago de Cuba, Cuba.

Fuentes, H. (1998). El modelo de actuación profesional: una propuesta viable para el diseño de la educación superior. Santiago de Cuba: CEES "Manuel F". Universidad de Oriente.

Fuentes, H. y Lucio, A. (2009). Formación por competencias en la concepción de la universidad humana cultural, una propuesta desde la Universidad Estatal de Bolívar. Guaranda, Venezuela.

Gallart, M. (2011). Escuela-Empresa: un vínculo difícil y necesario. Recuperado el 23 de marzo de 2011

García, J. (2000). El perfeccionamiento del proceso de resolución de los problemas profesionales en las disciplinas BE para CT en la carrera de ingeniería Mecánica. (Tesis doctoral inédita). Universidad de Camagüey, Camagüey, Cuba.

González, M. y otros (2003). Curriculum y formación profesional. La Habana: Centro de estudios para el perfeccionamiento de la Educación Superior. ISBN 959-261-106-8.

Guzón, A. y otros. (2011). Cataurito de herramientas para el desarrollo local. La Habana: Caminos.

Hernández, I. (2000). El Proceso Pedagógico Profesional: un abordaje teórico y metodológico. La Habana: ISPETP: "Héctor Alfredo Pineda" 
Jiménez, S. (2003). Fundamentos del diseño curricular en la Educación Técnica y Profesional. Recuperado el 6 de mayo de 2010

Kemmis, S. (1986). Seven Principles for Program Evaluation in Curriculum Development and Innovation, en B. R. Rouse (ed.): New Directions in Educational Evaluation. Lewes: The Falmer Press, p. 117-140.

León, M. (2003). Modelo para la integración escuela politécnica mundo laboral en la formación de profesionales de nivel medio. (Tesis doctoral inédita). ISP "Enrique J. Varona", La Habana, Cuba.

Lotti, A. (2014). La enseñanza práctica en un primer plano. Periódico Trabajadores. Lunes 8 de septiembre de 2014, p. 6-7.

Mena, J. (2003). La integración Escuela Politécnica - Empresa: Una propuesta metodológica para su desarrollo adecuado. (Tesis de maestría inédita). ISPETP” Héctor A. Pineda Zaldívar", La Habana, Cuba.

Mena, J. (2008).La vinculación a las entidades laborales: una forma para elevar la calificación científica técnica del profesor de la ETP. En Revista Electrónica del Instituto Superior Pedagógico "Rafael María de Mendive". Pinar del Rio, Cuba.

Mined. (2010). Resolución Ministerial No.165/2010. Planes y programas de estudio para la formación de técnicos medios de la rama Ferroviaria. La Habana: Ministerio de Educación.

Mined. (2011). Resolución Ministerial No.147/2011. Planes y programas de estudio para la formación de técnicos medios con nivel Medio Superior Profesional de la rama Ferroviaria. La Habana: Ministerio de Educación.

Patiño, M. (1988). Análisis de la práctica pre profesional en la formación de técnicos medios: vías y exigencias para evaluar su efectividad. (Tesis doctoral inédita). ICCP, La Habana, Cuba.

Patiño, M. y otros. (1996). El modelo de escuela politécnica cubana. La Habana: Centro de Estudios de Pedagogía Profesional.

Polo, A. (2012). Metodología para el microdiseño curricular de la asignatura Matemática, especialidad Electricidad de la ETP. (Tesis doctoral inédita). UCP “José Martî”, Camagüey, Cuba.

Rosental, M. y Ludin, P. (1981). Diccionario Filosófico. La Habana: Editora Política.

Thompson, D. (2009) La formación laboral del Bachiller Técnico en la especialidad de Agronomía. (Tesis doctoral inédita). ISP “José de la Luz y Caballero”, Holguín, Cuba.

Valdés, E. y Portuondo, R. (2000). Elementos de Teoría y diseño curricular. Camagüey: CECEDUC. Universidad de Camagüey. (2da versión). 